\title{
Le Centre d'Apprentissage de Langues : représentations, motivations et réalités
}

Peter Prince et Marie-Noëlle Olive

\section{OpenEdition}

\section{Journals}

Édition électronique

URL : http://journals.openedition.org/asp/1190

DOI : 10.4000/asp. 1190

ISBN : 978-2-8218-0394-7

ISSN : 2108-6354

Éditeur

Groupe d'étude et de recherche en anglais de spécialité

Édition imprimée

Date de publication : 1 novembre 2003

Pagination : 85-98

ISSN : 1246-8185

Référence électronique

Peter Prince et Marie-Noëlle Olive, « Le Centre d'Apprentissage de Langues : représentations, motivations et réalités », ASp [En ligne], 41-42 | 2003, mis en ligne le 27 avril 2010, consulté le 01 mai 2019. URL : http://journals.openedition.org/asp/1190 ; DOI : 10.4000/asp.1190

Ce document a été généré automatiquement le 1 mai 2019

Tous droits réservés 


\title{
Le Centre d'Apprentissage de Langues : représentations, motivations et réalités
}

\author{
Peter Prince et Marie-Noëlle Olive
}

\section{Introduction}

1 Le présent article a pour objectif d'offrir un aperçu de la réalité d'un Centre d'Apprentissage de Langues (CAL), en partant de l'idée que cette réalité dépend en partie des motivations des différents acteurs (personnels du CAL et apprenants) concernés par son fonctionnement. De manière générale, une théorie de la motivation cherche à expliquer les raisons pour lesquelles on entreprend ou poursuit telle action; il s'agit en l'occurrence d'analyser ce qui motive les comportements qui constituent la vie du CAL à un moment donné de son évolution. La forme que prend un CAL sur le plan de son organisation pédagogique résulte de la rencontre entre personnels et apprenants : suite à une réflexion pédagogique, les premiers proposent une certaine organisation que les apprenants expérimentent, le bilan de la rencontre pouvant mener à des modifications de l'organisation. Dans cette perspective, les représentations des uns et des autres jouent un rôle important. Elles se modifient en fonction d'une évaluation constante de la situation. Or la motivation s'appuie en permanence sur ces représentations.

2 Cette analyse ne peut prétendre à l'exhaustivité. Construite à partir de l'expérience d'un CAL spécifique, l'image qui ressort ne saisira pas la richesse ou la créativité de ce qui peut se faire ailleurs. Mais il est à espérer qu'elle mettra en relief des moments ou des processus communs à tout CAL au cours de son évolution. Quel que soit le CAL, il met en place une forme de médiation pédagogique, c'est-à-dire une manière d'organiser un environnement matériel et cognitif individualisé, qui va permettre aux apprenants de construire des représentations méthodologiques débouchant sur un apprentissage efficace. L'accent sera donc mis sur les processus qui régissent cette médiation. 
3 L'analyse prenant comme axe central la motivation, il convient d'abord de présenter la manière dont celle-ci est prise en compte sur le plan théorique avant de passer à l'étude des rapports qu'elle entretient avec l'organisation pédagogique. Ensuite nous présentons un exemple d'évaluation du CAL, tentative pour saisir une partie de la réalité d'une telle structure.

\section{Motivation et représentations}

4 La plupart des chercheurs qui travaillent sur la motivation admettent qu'elle implique un certain dosage de désirs et d'efforts en vue d'atteindre un but. Dès lors que l'on essaie de rendre compte d'un contexte spécifique donné, les composantes de la motivation s'avèrent plus complexes, mais cette définition peut servir dans un premier temps à une réflexion sur la motivation qui se manifeste dans un CAL. Si la définition citée s'applique aux apprenants, il suffit de modifier le but pour qu'elle s'applique aussi au personnel.

\subsection{La motivation du personnel}

5 Le but que le personnel cherche à atteindre est la création de conditions propices à l'apprentissage chez autrui. De nombreux facteurs peuvent influencer la motivation à atteindre ce but, dont certains se retrouvent, tout à fait logiquement, chez tous les enseignants. Pour Hackman et Oldham (1975), un travail est motivant dès lors qu'il est jugé important par autrui, requiert une variété de compétences, est identifiable comme un tout, permet une autonomie et donne lieu à des résultats perceptibles. Ces facteurs mènent à une forte motivation intrinsèque souvent constatée chez des enseignants. Mais si beaucoup d'enseignants se démotivent, c'est qu'il existe aussi des facteurs négatifs, dont Dörnyei (2001: 165) dresse une liste : le stress, les contraintes institutionnelles qui limitent l'autonomie, un sentiment d'inefficacité, la répétitivité du contenu, et le peu de perspectives de carrière. Par rapport à la salle de classe dans l'enseignement secondaire, où ces facteurs négatifs paraissent importants, il est probable qu'un CAL dans un établissement de l'enseignement supérieur offre un environnement de travail plus motivant. Il n'est pas impossible, cependant, qu'un sentiment d'inefficacité s'installe, voire une certaine forme de stress, dès lors que les ressources humaines nécessaires à répondre à la demande paraissent insuffisantes. Ce paramètre varie fortement selon les situations, et nous préférons examiner ici un facteur plus généralisé : que le CAL soit riche en ressources humaines ou démuni, la motivation de son personnel sera plus ou moins forte selon qu'ils estiment progresser vers le but visé ou non. Or, de par la nature de l'enseignement universitaire, souvent dispensé sur un seul semestre à d'importants groupes d'étudiants, le facteur motivant qui consiste à constater les résultats de son travail n'est pas toujours présent ; il est donc important de garder constamment à l'esprit le souci d'évaluer le dispositif mis en place.

6 Une discussion de cette question viendra en fin d'article, mais d'ores et déjà on peut souligner le rapport étroit entre évaluation et représentations, car on ne conclura à la réussite du dispositif que si l'on a une idée claire de ce en quoi cette réussite consiste. S'il paraît évident que la meilleure preuve de sa réussite serait le constat d'une progression de la compétence en langues chez les étudiants, encore faut-il savoir ce que cette compétence est censée recouvrir. Par ailleurs, un aspect central de tout CAL est le développement de l'autonomie ${ }^{1}$ chez les apprenants, ce qui peut également apporter une 
preuve de réussite à condition d'avoir une représentation de ce que ce terme signifie et de pouvoir la mesurer.

7 La motivation du personnel est beaucoup moins étudiée que celle des apprenants. Tout en soulignant le bénéfice qui viendrait d'un rééquilibrage de cette situation, on peut lui trouver une justification dans la mesure où l'apprenant est censé être le premier bénéficiaire de l'enseignement. Par ailleurs, même si la motivation du personnel influence celle des apprenants (Dörnyei 2001 : 35-37) son rapport avec l'apprentissage effectué est moins direct. Il convient donc de dégager quelques lignes conductrices qui peuvent déterminer le comportement d'un apprenant qui vient au CAL.

\subsection{Les représentations des apprenants}

8 Dörnyei \& Otto (1998) nous proposent un modèle dynamique de la motivation qu'ils séparent en trois phases. Dans un premier temps il faut déjà une motivation pour former une intention; ensuite d'autres formes de motivation sont nécessaires pour s'engager dans l'action et la poursuivre; enfin on évalue les résultats de l'action avant de décider si on souhaite la continuer ou non. Dans le cas d'étudiants en formation initiale, la première phase est le plus souvent occultée, car l'étude d'une langue leur est imposée. Cela rend d'autant plus nécessaire la création de conditions propices au maintien d'un niveau élevé de motivation lors des deux autres phases. Pour Deci \& Ryan (1985), plus les étudiants ont le sentiment qu'ils peuvent contrôler les conditions de leur apprentissage, plus ils seront motivés. Il leur apparaît donc que l'autonomie qu'ils trouvent dans un CAL est souvent le garant d'une bonne motivation au départ. Ce n'est pas suffisant, toutefois, car d'autres facteurs entrent en jeu, sous forme de représentations de la tâche à accomplir. En simplifiant quelque peu les mécanismes de la motivation, on peut dégager trois représentations qui seront déterminantes : le plaisir que procure une activité, sa facilité et sa pertinence. L'interaction entre ces représentations est complexe: si nous sommes naturellement attirés par des activités que nous ressentons comme agréables, celles-ci ne sont pas toujours les plus motivantes, car elles peuvent être perçues comme non pertinentes par rapport à l'objectif fixé. Par ailleurs la réussite n'aura un effet motivant que si la tâche accomplie comporte un certain niveau de difficulté, permettant à l'apprenant de lui accorder davantage de valeur. Ces représentations de la tâche sont activées par anticipation, avant de s'y engager, et réexaminées après, lors de la phase d'évaluation. La motivation durable nécessaire pour poursuivre un processus aussi long et laborieux que l'apprentissage d'une langue ne s'installera que si l'apprenant a la conviction que les activités entreprises, aussi agréables soient-elles, sont non seulement réussies mais sont aussi autant de jalons attestant d'un progrès réel vers un but qu'il considère comme important. Si certains enseignants arrivent à installer ce type de motivation, c'est en partie grâce aux activités qu'ils proposent, la façon dont ils les présentent et l'évaluation qu'ils fournissent à leurs élèves. Tous ces moments de l'enseignement sont organisés de manière très différente dans un CAL, où il s'agit de créer un espace de médiation pédagogique qui permet aux apprenants de se prendre en charge, tout en essayant de susciter un niveau élevé de motivation. La réussite d'une telle démarche passe par la modification d'un autre type de représentation, celle qui concerne le déroulement concret d'un cours de langues.

9 D’après Schank \& Abelson (1977), nombre de nos représentations mentales sont organisées sous forme de scripts, c'est à dire un enchaînement ordonné de 
représentations d'actions, d'actes de parole ou d'objets. Par exemple, dans le script « aller au restaurant ", il y aura des représentations d'actions telles que s'asseoir à une table, étudier la carte, commander des plats etc. Certains éléments sont considérés comme obligatoires (par exemple, payer), d'autres facultatifs (prendre un dessert ou du café). L'ordre des évènements est souvent relativement fixe, comme dans le cas d'un repas au restaurant, mais un script peut avoir de multiples variantes - prendre un repas dans un fast food entraîne plusieurs modifications au script du restaurant. De la même façon « aller au CAL » active des représentations très différentes de celles du script « aller en cours de langue ». Bien que l'apprentissage d'une langue n'ait comme élément obligatoire que le fait de s'engager dans une certaine forme d'activité cognitive, la pratique de cette activité dans une salle de classe pendant plusieurs années entraîne la formation d'un script où l'enseignant, avec toutes les pratiques pédagogiques qu'il met en œuvre, apparaît comme un élément obligatoire. D'où le désarroi de certains apprenants lorsqu'ils entrent au CAL pour la première fois et constatent non seulement un espace qui n'a rien à voir avec une salle de classe, mais aussi une profusion de supports et de matériaux et une absence de l'enseignant qui devrait être là pour leur donner du travail.

\subsection{Motivation et métacognition}

10 Après tant d'années où le choix, le déroulement et la durée des activités ont été imposés aux apprenants, cette liberté peut également être grisante. Ainsi, deux sentiments contradictoires peuvent coexister. Il n'est pas exclu qu'un étudiant passe plusieurs séances au CAL de manière agréable tout en ayant l'impression de ne pas avancer dans son apprentissage. L'absence de l'enseignant, de son intervention en tant que médiateur pédagogique, déstabilise l'étudiant. Le choix de l'activité, la présentation des consignes, la vérification de son bon déroulement et l'évaluation des performances nécessitent une réflexion approfondie. Ces temps forts sont habituellement pris en charge par le professeur, qui grâce à sa formation et son expérience devient expert en la matière. Cette prise en charge étant presque invisible pour les élèves, elle ne rentre même pas dans le script, qui devra être fondamentalement remodelé dans le CAL, celui-ci - pour filer la comparaison alimentaire - ressemblant plus à un buffet qu'à un restaurant. La compétence à exercer, le support à utiliser, ne sont pas présentés dans un ordre défini, et l'apprenant à qui l'on ne donne pas des critères de choix peut se trouver dans l'embarras. Il existe ainsi une relation entre les représentations du déroulement d'une séance de travail et celles qui jouent sur la motivation : si l'apprenant n'arrive pas à entamer une réflexion métacognitive, lui permettant de définir un parcours pédagogique, il est possible que les activités qu'il entreprendra ne lui paraissent pas suffisamment pertinentes pour que sa motivation se maintienne. Il est à noter que le constat de la non pertinence d'une activité reflète déjà une certaine réflexion métacognitive, puisqu'on ne peut faire un tel constat que si on a, d'une part, conscience d'un objectif à atteindre et d'autre part une manière d'évaluer ses progrès. En l'absence de cette réflexion, il est possible de passer un semestre entier au CAL sur des activités à la fois agréables et faciles sans forcément se démotiver, parce qu'on n'a pas d'objectif. Ce cas de figure est plutôt rare : si la fréquentation du CAL est soumise à une évaluation qui donne lieu à des crédits pour les étudiants, l'épreuve d'évaluation constitue par définition un objectif. La pertinence des activités devient alors un facteur important dans la motivation. Dans la mesure où le CAL vise à créer des conditions propices à l'apprentissage, un de ses devoirs est donc de rendre visible la médiation pédagogique de façon à ce que les apprenants 
puissent s'approprier une démarche d'apprentissage, sous forme de réflexion métacognitive, tout en gardant l'autonomie qui est au cœur du projet du CAL. Si la nécessité de passer une épreuve en fin de semestre peut contraindre beaucoup d'étudiants à se fixer des objectifs, elle ne dispense pas le CAL de mettre en place un dispositif visant à susciter une motivation intrinsèque. L'évaluation ne va pas forcément à l'encontre de ce type de motivation, puisque l'apprenant peut parfaitement adhérer à la nécessité d'être évalué, mais elle favorise davantage une motivation extrinsèque, ce qui amène certains apprenants, une fois l'évaluation passée, à ne plus soutenir leurs efforts.

\section{L'organisation pédagogique d'un centre de langues}

\subsection{La présentation du centre}

11 Le défi auquel est confronté un CAL est de rendre accessible, sur le plan matériel et intellectuel, une certaine forme de réflexion pédagogique, ainsi que des éléments théoriques issus de la didactique des langues. Le CAL est un lieu de rencontre où se côtoient la réflexion des personnels et celle des utilisateurs, qui arrivent avec des pratiques et des attitudes héritées du passé. Ces derniers ne seront pas forcément immédiatement réceptifs à un discours les incitant à se lancer dans une réflexion métacognitive sur la manière d'apprendre une langue. C'est pour cette raison que ces éléments de médiation pédagogique doivent être disponibles en permanence: bien qu'une séance d'introduction, au cours de laquelle un responsable du CAL expliquera son fonctionnement, soit sans doute nécessaire, elle n'est pas suffisante car la partie du discours consacrée aux principes pédagogiques qui régissent le centre n'est pas assimilée par tous.

Une présentation du centre sur ordinateur peut en partie pallier ce problème, car elle est disponible en permanence. Elle peut facilement prendre en charge tout ce qui concerne le fonctionnement administratif et la disposition matérielle du centre. Ceci n'est pas négligeable puisque ces éléments vont nécessairement faire partie du script que les étudiants auront à construire. Plus vite ils sauront s'orienter dans le centre, plus vite ils pourront se consacrer à l'apprentissage. Par ailleurs, une telle présentation peut en partie dégager le personnel de la nécessité de répéter des informations, le libérant ainsi pour d'autres tâches. Mais que la présentation initiale du centre soit faite sur ordinateur ou de vive voix, elle ne pourra pas, à elle seule, remplir l'objectif qui est de permettre aux apprenants de gérer leur autonomie efficacement et le plus rapidement possible. D'où la nécessité d'autres formes de présentation qui dépassent l'aspect matériel pour constituer le dispositif de médiation pédagogique dont de nombreux apprenants auront besoin au bout de quelque temps. Pour ce faire, un CAL peut faire appel à plusieurs méthodes. Par exemple, un questionnaire peut inciter l'apprenant à réfléchir sur ses objectifs (Oxford 1990 : 179) ou sur le fonctionnement même du CAL (Gardner \& Miller 1999 : 176). Souvent une fiche de suivi, remplie à la fin de chaque séance, fait également partie de la panoplie d'outils que propose le CAL (un exemple est proposé en annexe). Cette fiche permet à l'apprenant, tout comme au personnel, d'avoir une vision rapide du parcours entrepris. Autre possibilité, introduite récemment de manière limitée dans notre $\mathrm{CAL}$, des fiches méthodologiques et des séances de tutorat font l'objet d'une réflexion en cours dont nous présentons ici quelques éléments. 


\subsection{L'aide méthodologique}

13 Les fiches méthodologiques, rédigées en français, décomposent l'acte d'apprentissage, le rendant assimilable élément par élément. Il ne s'agit pas de diriger l'apprenant dans son parcours, mais de lui proposer des conseils ou des informations pouvant l'aider dans ses choix. Les domaines couverts par ces fiches sont potentiellement vastes: les quatre compétences, les façons d'améliorer ses connaissances lexicales ou grammaticales, la planification des activités, le travail collaboratif, l'auto-évaluation. En ce qui concerne la planification, une possibilité est de proposer un plan d'étude ou de suggérer un parcours, ce qui peut rassurer l'apprenant mais comporte aussi le risque qu'il n'explore pas pleinement les possibilités que lui offre l'autonomie.

14 La rédaction de fiches méthodologiques n'est pas facile, car elles doivent non seulement être claires et concises mais aussi convaincantes, et comporter conseils et explications. L'obligation de concision entraîne un compromis car trop d'informations, difficiles à assimiler, ne favoriseront pas la prise de conscience recherchée. Il est possible toutefois de rédiger des fiches avec des niveaux de détail différents, allant du plus général (par exemple une fiche sur la planification du parcours d'apprentissage), des fiches à un niveau plus spécifique traitant de chaque compétence (une explication simple: dire en quoi elle consiste, une indication des objectifs qui peuvent être fixés), enfin des fiches détaillées sur les différentes activités. Ces dernières indiqueront quels supports sont adaptés pour chaque type d'activité et donneront, le cas échéant, des consignes. Disponibles en permanence, ces fiches représentent une manière parmi d'autres de permettre à l'apprenant de construire une représentation cohérente de ce que signifie apprendre une langue dans un CAL. Il est peu probable, ni profitable, qu'elles soient lues en une seule fois, mais elles peuvent servir à illustrer ou étayer des conseils que viendrait chercher un apprenant lors d'une séance de tutorat.

Il est clair que le choix des fiches méthodologiques proposées, ainsi que leur contenu, relève du personnel du CAL et traduit une réflexion pédagogique qui lui est propre. Ainsi ces fiches peuvent varier beaucoup d'un CAL à un autre, ou bien en être totalement absentes; mais, en tout état de cause, une certaine forme d'accompagnement méthodologique s'impose. L'autonomie ne consiste pas à laisser l'apprenant se débrouiller tout seul (Little $1991: 3$ ).

Le tutorat représente un deuxième aspect de la médiation pédagogique, sans doute le plus significatif dans la mesure où cette séquence intègre la richesse du dialogue entre deux personnes (ou davantage). Mozzon-McPherson (2000) souligne que le tuteur dans un CAL n'est pas un enseignant mais un conseiller. Même si par ailleurs il peut être enseignant de langues devant une classe, son rôle au CAL est de faciliter l'apprentissage en amenant l'étudiant à une prise de conscience méthodologique et en le guidant vers des ressources appropriées. Pour Mozzon-McPherson, ce travail représente un nouveau métier. S'il est effectué par des enseignants, ils auront besoin d'une formation spécifique. Cette formation est d'autant plus nécessaire que les apprenants eux-mêmes risquent d'arriver à la séance de tutorat avec une représentation erronée du nouveau rôle joué par l'enseignant. Celui-ci ne joue plus le rôle de l'enseignant classique. Il faudra amener les apprenants à modifier cette représentation. 


\subsection{Stratégies et objectifs}

17 Ces différentes formes de médiation nécessitent chez les apprenants de nouvelles stratégies d'apprentissage, ainsi que l'émergence de nouveaux comportements. Une définition d'objectifs s'impose en début de parcours, avec une prise de conscience métacognitive. La «littérature" sur ces stratégies est à ce jour abondante (voir par exemple Oxford 1990; O'Malley \& Chamot 1990; Cohen 1998). Comme le remarque McDonough (1999), une compréhension fine de la façon dont elles sont agencées et utilisées reste encore à trouver; par ailleurs il semble qu'une formation spécifique à l'utilisation de stratégies ne soit bénéfique que dans certaines circonstances (Benson 2001 : 144-146). Ces réserves ne mettent pas en cause toutefois la conclusion plus générale sur le rôle primordial de la métacognition dans l'apprentissage d'une langue (Wenden 1998).

Dans la mesure où l'on considère qu'une stratégie, pour mériter cette appellation, doit être consciente (Cohen 1988 : 11), elle est liée à la notion d'objectif, car elle représente un moyen de l'atteindre. Or dans une étude sur les techniques motivationnelles menée auprès de 200 enseignants de langues en Hongrie, Dörnyei \& Csizér (1998) ont trouvé que l'incitation à une prise de conscience des objectifs (goal orientedness) était une des techniques les moins utilisées. En d'autres termes, l'enseignant sait où il veut amener ses élèves, mais il ne rend pas l'objectif explicite, pas plus qu'il explique toujours les raisons pour lesquelles il propose telle ou telle activité dans la classe. Oxford \& Shearin (1994) soulignent pourtant à quel point un comportement orienté vers des objectifs peut avoir un effet motivant, et l'autonomie inhérente à l'apprentissage dans un CAL rend cet aspect particulièrement important. En effet, en l'absence d'une représentation suffisamment précise d'un objectif à atteindre, l'apprenant dans un CAL risque de faire un usage peu efficace de l'autonomie dont il jouit. La médiation pédagogique mise en place vise donc non seulement à faire adopter des stratégies, mais aussi à faire prendre conscience de la manière dont ces stratégies contribuent à des objectifs à plus long terme.

\section{L'évaluation d'un centre de langues}

\subsection{Les types d'évaluation}

Si des termes clé jusqu'ici ont pu être: médiation, représentation et motivation, c'est parce qu'ils recouvrent des dimensions présentes dans tous les centres de langues, comme dans toute situation d'enseignement. Ces dimensions ont une influence majeure sur la réussite ou l'échec de la formation. Mais une réflexion sur ces aspects, aussi utile soit-elle, ne permet pas pour autant de constater, encore moins de mesurer la réussite. Le CAL se compose de réalités observables ou quantifiables - l'agencement spatial, la facilité d'accès aux ressources, le nombre d'usagers, le degré de convivialité de l'endroit - mais la véritable réalité d'un CAL est constituée par les actes d'apprentissage entrepris et les gains qui en résultent. Ces gains s'expriment en termes de connaissances, de compétences, de confiance ou de motivation. Cette réalité peut être mesurée sous forme d'une évaluation qui, au-delà des objectifs des apprenants, permet au personnel de voir si leur propre objectif - la mise en place d'un dispositif propice à l'apprentissage - est en voie d'être atteint. Quelle que soit la forme qu'elle prend, cette évaluation équivaut au 
questionnement de sa propre pratique auquel se livre chaque enseignant, pour peu qu'il possède une motivation intrinsèque qui l'amène à chercher sans cesse une pédagogie plus adaptée ou plus efficace.

Par ailleurs, comme le remarquent Gardner \& Miller (1999 : 226), une évaluation du CAL peut être nécessaire à des fins de bilan, permettant de rendre compte à d'autres instances de l'institution de son efficacité, ou de justifier une demande de ressources supplémentaires. Gardner \& Miller séparent l'efficacité en deux versants : effectiveness, mesuré en termes d'apprentissage, et efficiency, qui prend aussi en compte les ressources déployées pour arriver à tel résultat. Bien que la culture universitaire française n'incite pas, jusqu'ici, les composantes de l'établissement à se soumettre à des évaluations rigoureuses de ce type, elles peuvent s'avérer utiles dans le cas d'un CAL dont le rôle est méconnu, voire mis en cause, par des collègues habitués à un enseignement plus traditionnel. Gardner \& Miller remarquent très justement, toutefois, que plus une évaluation est complète, plus elle est difficile à mener et perturbe la vie du centre. Si l'évaluation est effectuée par le personnel, ce qui est le plus souvent le cas, il est peu probable que celui-ci dispose du temps nécessaire à une évaluation approfondie. Il faut donc opérer des choix.

\subsection{Une évaluation quantitative}

21 L'évaluation décrite ici, effectuée en juin 2003, consistait en une estimation quantitative des ressources utilisées et des activités entreprises. Il est à souligner que l'image d'une certaine réalité qui en ressort ne concerne qu'un CAL spécifique et n'a de valeur générale que dans la mesure où d'autres CAL ont des objectifs ou des démarches semblables.

L'objectif était de dresser un bilan des ressources utilisées dans le CAL afin d'avoir une vision globale des activités entreprises et d'apporter des modifications dans le cas où certaines ressources seraient sous-utilisées. Le travail effectué par 190 étudiants, répartis en quatre filières ( $1^{\text {re }}$ année psychologie, $\mathrm{n}=80 ; 1^{\text {re }}$ et $2^{\mathrm{e}}$ année géographie, $\mathrm{n}=21$; licence information-communication, $\mathrm{n}=38$; licence pluridisciplinaire, $\mathrm{n}=51$ ), et fréquentant le CAL pour étudier l'anglais, a été analysé grâce à des informations en provenance de trois sources: les réponses des étudiants à un questionnaire comprenant une partie de libres commentaires incluant la critique éventuelle de la formation proposée; un entretien individuel de 7 à 10 minutes en français, consacré au parcours entrepris pendant le semestre (cet entretien donne lieu à une évaluation chiffrée, laquelle constitue un quart de la note finale en langue donnée aux étudiants) ; l'étude de la fiche de suivi (voir annexe).

\subsubsection{Les ressources utilisées}

Le tableau 1 indique les pourcentages d'étudiants ayant utilisé, au moins une fois dans le semestre, chacune des 11 ressources proposées. 
Tableau 1. Étudiants ayant utilisé les ressources

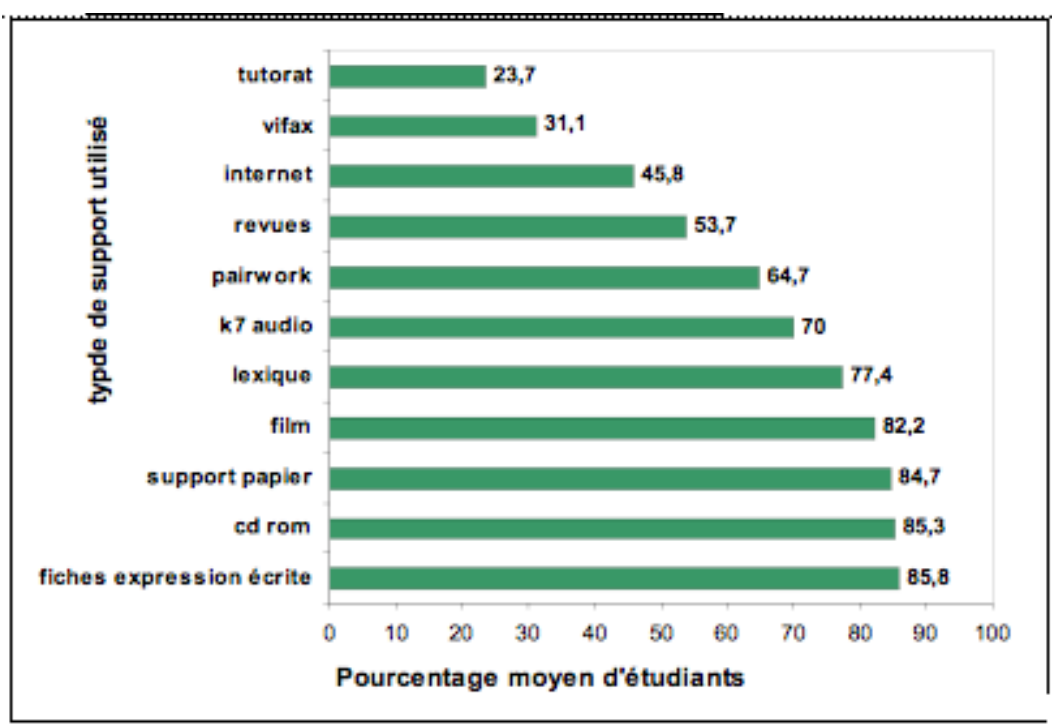
d'un quart des étudiants ont demandé à rencontrer un enseignant. Les autres supports (décrits sur le graphique) ont un taux d'utilisation élevé, sauf le système Vifax (actualités enregistrées sur cassette vidéo, accompagnées de questions de compréhension de l'oral), dont tout le potentiel en tant que ressource n'a pas été suffisamment mis en avant. De nombreux étudiants ont travaillé l'expression orale en binôme à partir de matériel proposé dans des classeurs avec des consignes (pairwork). Ceci est une source de satisfaction, dans la mesure où ces activités ne sont proposées que depuis deux ans. La première année, faute de présentation claire ou convaincante, très peu de personnes les ont utilisées.

L'entretien individuel a permis d'apporter des éclairages qualitatifs supplémentaires. En ce qui concerne le tutorat, plusieurs raisons peuvent être invoquées pour expliquer le faible taux de recours à ce dispositif. La première semble être une perception floue du rôle que le tuteur est censé jouer. Dans la mesure où le personnel, tuteurs ou non, est souvent présent pour répondre à des questions ponctuelles, l'intérêt de prendre un rendez-vous pour un examen plus détaillé de leurs parcours ne paraît pas évident aux étudiants, et ceci malgré des fiches méthodologiques expliquant le rôle du tutorat. Certains avouent une certaine timidité et n'ont pas toujours envie de dévoiler leur ignorance. Il est clair par ailleurs que bon nombre d'étudiants n'ont effectivement pas besoin de ce type d'assistance, puisqu'ils profitent déjà pleinement de l'autonomie qui leur est offerte. Enfin une dernière raison est le manque de personnel qualifié à certains moments, les tuteurs n'étant présents que pendant des créneaux horaires qui ne conviennent pas toujours aux étudiants.

Les fiches méthodologiques ne figurent pas parmi les ressources citées car les étudiants n'indiquent pas sur leurs fiches de suivi s'ils les ont consultées, ceci sans doute parce qu'elles ne comportent pas de contenu dans la langue cible. Par ailleurs, s'ils peuvent emporter les fiches avec eux, rien ne garantit qu'elles seront soumises à une lecture attentive par la suite. Selon les informations recueillies à l'entretien, il s'avère qu'elles sont peu prises en compte, soit elles ne sont pas consultées du tout, soit elles sont lues rapidement. Les étudiants, pressés peut-être de travailler avec des supports considérés 
comme plus attrayants, ou plus pertinents parce qu'en langue cible, réfléchissent peu à leur contenu. La portée de ces fiches semble donc limitée en dehors des cas où, lors d'une séance de tutorat, un tuteur peut expliciter davantage l'intérêt qu'elles ont. Il faut noter toutefois que, comme pour le tutorat, beaucoup d'étudiants arrivent à naviguer dans le CAL et à établir un parcours cohérent sans avoir besoin de consulter des fiches; il n'en reste pas moins que ces étudiants, tout en faisant preuve d'une autonomie satisfaisante (à la fois de leur propre point de vue et de celui de l'évaluateur du CAL), pourraient en profiter encore davantage s'ils entamaient une réflexion méthodologique approfondie. Le dispositif d'incitation à cette réflexion peut sans doute être amélioré.

Il existe une réelle difficulté à mesurer un comportement autonome. Que signifie un niveau 'satisfaisant'? En l'absence d'un instrument de mesure fin on se contente d'approximations. Il suffit parfois de regarder une fiche de suivi, ou d'observer un comportement in situ, pour constater que l'apprenant a parfaitement compris comment gérer son apprentissage. A l'opposé, il est parfois évident que l'apprenant se sent perdu. Entre ces deux extrêmes, bon nombre d'apprenants semblent naviguer dans le CAL sans difficulté, mais sans non plus avoir entrepris une réflexion sur leur parcours. Or comme le remarque Benson (2001 : 98)

Learners who are asked to take greater control of their learning, or who are forced by circumstances to do so, may be capable of performing the actions involved in self-management of learning, but they will not necessarily possess the cognitive capacities that will make these actions systematic or effective.

Il serait sans doute irréaliste d'espérer que tous les apprenants développent ces capacités cognitives, mais il est du devoir du CAL de mettre tout en œuvre pour les y inciter.

\subsubsection{Indicateurs de la motivation}

Comme le souligne Dörnyei $(2001: 185)$ la motivation n'est pas directement observable. Elle n'est discernable qu'à travers des comportements ou des impressions subjectives exprimées par les apprenants. Parmi les premiers, nous comptons ici le nombre de productions écrites des étudiants. En effet, les films, les fiches d'apprentissage du lexique, les cassettes de compréhension orale et les activités d'expression orale sont accompagnés de fiches destinées à susciter des quantités diverses d'expression écrite, allant de réponses courtes à la rédaction d'un ou de plusieurs paragraphes. Si ces productions ont un caractère plus ou moins obligatoire, soit parce qu'elles font partie intégrante de l'activité, soit parce qu'elles sont fortement recommandées, il est tout à fait possible aux étudiants de s'y dérober si l'effort qu'elles impliquent leur semble trop important ou inutile. On obtient par ailleurs une idée du niveau de motivation à travers un autre type d'écrit, entièrement facultatif, à savoir la prise de notes suite à la consultation d'ouvrages de référence. Parmi ces fiches personnelles, nous avons seulement pris en compte celles consacrées à des points de grammaire, car le vocabulaire a été associé aux fiches « lexique ». Le tableau 2 indique, toutes filières et tous types de productions confondus, le nombre de ces traces écrites.

Tableau 2. Productions écrites des étudiants

\begin{tabular}{|c|c|c|c|c|c|c|}
\hline Nombre de productions & 0 & $1-5$ & $6-10$ & $11-15$ & $16-20$ & $>20$ \\
\hline \% d'étudiants & 15,3 & 41,1 & 26,2 & 10,5 & 3,2 & 3,7 \\
\hline
\end{tabular}


Toutes filières confondues, on obtient une moyenne générale de 7,8 productions écrites par étudiant, ce qui peut paraître peu pour une moyenne de 30 heures passées au CAL. Il faut noter qu'en tant que compétence à améliorer, l'expression écrite ne figure pas parmi les objectifs prioritaires des étudiants. Il n'occupe pas une place importante dans l'épreuve de langues à la fin du semestre. En tant que support d'une mémorisation ou d'une révision ultérieure, en revanche, l'écrit joue un rôle important. Dans un CAL, contrairement à une salle de classe où l'enseignant peut ménager des phases spécifiquement consacrées à l'écrit, cet effort de prises de notes est laissé à la discrétion de l'apprenant. Dans un cours traditionnel l'étudiant dispose sans doute de davantage de traces écrites, d'autant plus que certaines ressources très utilisées dans le CAL (Cédérom) ne donnent lieu qu'à des traces écrites éphémères. Il est moins sûr, toutefois, que dans une salle de classe les traces écrites résultent autant de la propre initiative de l'étudiant. Dans les deux cas, il convient de souligner que l'effet réel produit par la prise de notes sur l'apprentissage reste méconnu.

31 Sans doute une des meilleures indications de la motivation est le nombre d'heures passées dans le CAL. Contrairement à la salle de classe, où le nombre d'heures de cours est fixe, le CAL offre la possibilité de dépasser le nombre d'heures de présence demandées pour satisfaire aux obligations de la formation. Or 58\% des étudiants ont effectivement dépassé ce nombre d'heures, la plupart de peu, certes, mais on peut estimer que ce chiffre est plutôt encourageant dans la mesure où il s'agit d'un public pour qui l'apprentissage d'une langue est en concurrence avec des matières souvent jugées plus importantes. A l'inverse, un tiers des étudiants sont restés en dessous du nombre d'heures demandées, ce qui indique que le CAL n'a pas réussi à les (re)motiver. Parmi ce tiers, il faut noter l'existence de deux types d'apprenants : ceux qui ont déjà un niveau en langues qui leur permet de s'absenter sans craindre un échec à l'épreuve de fin de semestre, et un groupe dont les raisons sont liées au peu d'importance qu'ils accordent à l'objectif affiché.

\subsection{Une évaluation des apprentissages}

L'étude statistique qui ressort de cette évaluation permet au personnel d'avoir une meilleure idée du comportement des étudiants. Le dispositif du CAL peut ainsi être modifié afin de rendre son fonctionnement plus clair, d'améliorer l'accessibilité de certaines ressources, et de favoriser l'émergence de nouvelles stratégies d'apprentissage. La question demeure, cependant, de savoir si les conditions mises en place dans un CAL mènent effectivement à un apprentissage réel.

Dans une étude précédente conduite dans le même CAL (Prince 2000), cette question a trouvé un début de réponse dans une comparaison des progrès effectués par deux groupes d'étudiants. Un fréquentait le centre de langues (groupe CAL), l'autre recevait un enseignement traditionnel (groupe classe). Un test a été proposé à chacun des groupes, en début et en fin d'expérimentation. La tâche consistait à transcrire en anglais le contenu d'un texte entendu deux fois. Le premier groupe a vu son taux d'erreurs morphosyntaxiques baisser significativement entre le début et la fin de l'expérimentation alors que pour le deuxième groupe le taux restait sensiblement le même. Une autre mesure, portant sur le nombre d'unités sémantiques correctement identifiées, révélait une légère progression pour le groupe CAL et une régression pour l'autre groupe. Enfin, le groupe CAL a également devancé l'autre, mais de manière non significative, dans une tâche de production orale où la mesure portait sur l'aisance d'expression, évaluée en nombre de 
syllabes utiles (appartenant à des mots qui contribuent à l'élaboration du message) par seconde.

Une telle comparaison est à prendre avec beaucoup de précaution: les objectifs spécifiques et le contenu des formations n'étaient pas les mêmes pour les deux groupes. Par ailleurs, l'étude n'a de valeur que par rapport à la situation spécifique des deux groupes, et ne prétend pas comparer le concept général de l'auto-formation guidée par rapport à l'enseignement présentiel. Malgré ces réserves, la comparaison garde son intérêt dans la mesure où les tâches expérimentales mettaient en évidence des compétences générales et non pas des connaissances liées à un programme spécifique. Ces compétences (compréhension de l'oral, expression orale) figuraient parmi les objectifs généraux liés aux deux types de formation. Même si le groupe classe passait plus de temps à lire des textes (l'évaluation finale consistait en une synthèse de textes), l'enseignement était dispensé en anglais, et les étudiants étaient encouragés à s'exprimer. Par ailleurs les réponses à un questionnaire révélaient des différences entre les groupes lorsqu'ils jugeaient eux-mêmes les progrès accomplis, avec les gains pour le groupe CAL étant significativement supérieurs pour toutes les compétences sauf la lecture. Pour celleci, les gains estimés par le groupe CAL restaient néanmoins légèrement supérieurs à ceux du groupe classe, malgré l'importance accordée à la lecture pendant la formation de ce dernier. Par ailleurs, le groupe CAL estimait, significativement plus que le groupe classe, avoir gagné en confiance, motivation et prise de conscience de stratégies d'apprentissage. En revanche, le groupe CAL exprimait, significativement plus que le groupe classe, le souhait de recevoir une formation plus structurée.

Si une telle étude peut conforter l'idée que l'auto-formation guidée est une voie intéressante à développer, elle ne dispense pas du besoin d'en rechercher constamment les meilleures conditions. Il semble ainsi plus profitable que la recherche désormais porte sur ces conditions que sur des comparaisons avec l'enseignement en classe de langues. Non pas que celui-ci soit nécessairement inférieur car tout dépend des conditions de son exercice (nombre d'étudiants, motivation de l'enseignant), mais les centres de langues sont de toute façon installés en tant que structure reconnue et il ne s'agit pas de les remettre en cause mais de savoir jusqu'où leur rôle peut s'étendre et quelle politique il faut établir pour que l'efficacité soit au rendez-vous. Quelle que soit cette politique, le défi pour un CAL consiste à trouver une organisation où l'autonomie n'est pas perçue par les apprenants comme le résultat de l'abandon par les enseignants de leurs responsabilités, mais comme une occasion offerte aux apprenants de se prendre en charge et d'être pleinement responsables d'eux-mêmes.

\section{Conclusion}

La réalité d'un CAL que nous avons tenté de dégager dans cet article résulte de l'interaction entre représentations et motivations du personnel et des apprenants, donnant lieu pour les premiers à des actions entreprises pour créer un espace, à la fois matériel et cognitif, qui favorise l'apprentissage, et pour les seconds à des stratégies pour occuper cet espace. L'analyse s'est attachée ainsi à un niveau que l'on peut qualifier d'interne, où l'on cherche à élucider comment un CAL joue son rôle de lieu d'apprentissage. Il existe une autre réalité, toute aussi empreinte de représentations et de motivations, mais qui concerne la manière dont les centres de langues sont vus de l'extérieur, notamment par ceux qui ont la charge de mettre en place ou de conduire une 
politique des langues, que ce soit au niveau de l'établissement, de la région ou du pays. Une analyse de cette politique sortirait du cadre de cet article, mais on peut supposer que si les CAL existent, c'est parce qu'ils sont considérés, entre autres, comme offrant une réponse possible à un problème, à savoir :

la nécessité qui s'impose de surmonter les handicaps et de combler les retards accumulés de façon à ce que tous les étudiants, quelle que soit leur spécialité, quelle que soit leur formation, soient réellement préparés à vivre et travailler dans les conditions de communication, d'ouverture internationale, d'échanges linguistiques et culturels qui caractérisent le monde contemporain ${ }^{2}$.

La contribution réelle de chaque CAL à cette réponse au problème posé dépend de la situation locale et aux choix qui ont présidé à sa création et à son développement. $\mathrm{Si}$ l'auto-formation guidée a effectivement le potentiel d'apporter ne serait-ce qu'une partie de la réponse, il serait regrettable qu'une fois créé, le CAL soit amené non pas à prendre sa place dans la politique des langues de l'établissement mais à confronter, voire conforter, l'absence d'une telle politique. Mais quelles que soient sa taille et sa situation, un centre de langues ne peut apporter plus que ce que ses moyens humains et matériels lui permettent. Une véritable réussite se mesure donc à l'aune de sa réalité interne.

\section{BIBLIOGRAPHIE}

Cohen, A. 1998. Strategies in Learning and Using a Second Language. Harlow : Longman.

Deci, E. \& R. Ryan. 1985. Intrinsic Motivation and Self-Determination in Human Behavior. New York : Plenum.

Dörnyei, Z. 2001. Teaching and Researching Motivation. Harlow : Longman.

Dörnyei, Z. \& K. Csizér. 1998. « Ten commandments for motivating language learners: Results of an empirical study ». Language Teaching Research 2, 203-229.

Dörnyei, Z. \& I. Ootto. 1998. « Motivation in action: A process model of L2 motivation ». Working Papers in Applied Linguistics, Thames Valley University, London 4, 43-69.

Gardner, D. \& L. Miller. 1999. Establishing Self-Access: From Theory to Practice. Cambridge : Cambridge University Press.

Hackman, J. \& G. Oldham. 1975. « Development of the job diagnostic survey. » Journal of Applied Psychology 60/ 2, 159-170.

Little, D. 1991. Learner Autonomy 1: Definitions, Issues and Problems. Dublin : Authentik.

McDonough, S. 1999. « A hierarchy of strategies? ». In S. Cotterall \& D. Crabbe (eds.), Learner Autonomy in Language Learning: Defining the Field and Effecting Change. Frankfurt : Peter Lang.

Mozzon-McPherson, M. 2000. « An analysis of the skills and functions of language learning advisers ». Links \& Letters 7, (Autonomy in Second Language Learning, Universitat Autonoma de Barcelona), 111-126. 
O’Malley, J. \& A. U. Chamot. 1990. Learning Strategies in Second Language Acquisition. Cambridge : Cambridge University Press.

Oxford, R. 1990. Language Learning Strategies: What Every Teacher Should Know. Boston : Heinle \& Heinle.

Oxford, R. \& J. Shearin. 1994. « Language learning motivation: Expanding the theoretical framework ». Modern Language Journal 78, 12-28.

Prince, P. 2000. « What is learnt and how? An examination of factors involved in the assessment of self-access language learning ». Communication présentée au Colloque English for Specific Purposes and Information Technology, 22-23 septembre 2000, University of Macedonia, Thessaloniki.

Schank, R. \& R. Abelson. 1977. Scripts, Plans, Goals and Understanding. Hillsdale, NJ : Lawrence Erlbaum.

Wenden, A. 1998. « Metacognitive knowledge and language learning ». Applied Linguistics 19/4, 515-537.

\section{ANNEXES}

\section{FEUILLE DE SUIVI Année 2003 / 2004}

Nom: Année du cursus $\left(1^{\mathrm{re}}, 2^{\mathrm{e}}\right.$, licence...) :

Prénom: Discipline (pyscho, géo, bio, histoire...) :

Tél.: Diplôme préparé au CALUP (TOEIC, TOEFL...):

Adresse: Langue choisie au CALUP :

Profession (si non étudiant):

Individual Study Plan:

1) Quels sont mes objectifs pour les semaines à venir?

2) Quels points dois-je améliorer ? (reading, exam practice, writing, oral expression, oral comprehension, grammar, vocabulary):

3) Y-a-t-il des sujets particuliers sur lesquels je désire travailler en rapport avec ma filière?

4) Après une découverte des outils du CALUP, quels matériels vais-je utiliser?

5) Quelles activités vais-je privilégier?

6) Comment évaluer mon travail ?

\begin{tabular}{|c|c|c|c|c|c|}
\hline \multicolumn{5}{|c|}{ Travail Accompli } \\
\hline Date & Durée & $\begin{array}{c}\text { Domaine } \\
*\end{array}$ & Titre du doc. & Contenu & $\begin{array}{c}\text { Utilité } \\
* *\end{array}$ \\
\hline
\end{tabular}




\begin{tabular}{|c|c|c|c|c|c|}
\hline 29.09 .03 & $\begin{array}{l}1 \mathrm{~h} 10 \\
45 \mathrm{~min} \\
30 \mathrm{~min} \\
30 \mathrm{~min}\end{array}$ & $\begin{array}{l}\text { G } \\
\text { C.o. } \\
\text { E.o. } \\
\text { Voc. }\end{array}$ & $\begin{array}{l}\text { English Plus CD } 1 \text { niv. } \\
3 \\
\text { Idem } \\
\text { Pairwork } n^{\circ} 4, \text { niv. } \\
\text { Inter. } \\
\text { Vocabulary list }\end{array}$ & $\begin{array}{l}\text { Passive voice, present } \\
\text { perfect } \\
\text { Radio and news } \\
\text { Likes and dislikes } \\
20 \text { mots appris }\end{array}$ & $\mid \begin{array}{l}4 \\
3 \\
5 \\
5\end{array}$ \\
\hline & & & & & \\
\hline & & & & & \\
\hline & & & & & \\
\hline
\end{tabular}

* Domaine : expression orale $=\mathrm{EO}$, expre. écrite $=\mathrm{EE}$, compréhension orale $=\mathrm{CO}$, comp. écrite $=\mathrm{CE}$, vocabulaire $=\mathrm{Voc}$, Grammaire $=\mathrm{G}$

** Utilité : 0 = activité inutile pour mes objectifs.... 5 = activité très utile pour mes objectifs

\section{NOTES}

1. La notion d'autonomie est complexe. Benson (2001) cite la définition de Holec dans son rapport au Conseil de l'Europe en 1981, où l'autonomie est considérée comme la capacité à prendre en charge son apprentissage. Pour Benson, cette capacité peut s'exercer en trois domaines interdépendants: la gestion de l'apprentissage (planification, stratégies), les processus cognitifs (attention, élaboration d'une réflexion métacognitive) et le choix du contenu à apprendre.

2. Rapport de la Commission pour la formation en langues des étudiants spécialistes d'autres disciplines, juillet 1998.

\section{RÉSUMÉS}

$\mathrm{Au}$ delà de son aspect matériel, la réalité d'un centre de langues est composée d'un 'espace' cognitif destiné à favoriser l'apprentissage. Les apprenants évoluent dans cet espace de manière plus ou moins autonome, le personnel du centre étant là pour les guider le cas échéant. Afin de trouver une organisation optimale, le personnel tente de prendre en compte les représentations et les motivations des étudiants, pour qui l'apprentissage dans un centre de langues est souvent une expérience nouvelle. Une évaluation du centre est nécessaire pour savoir comment ses ressources sont perçues et utilisées. Le dispositif mis en place peut ensuite être modifié pour favoriser l'émergence de stratégies propices à l'apprentissage. Nous présentons ici un exemple de cette réalité « interne » d'un centre de langues, où l'espace créé résulte de la rencontre entre réflexion didactique, supports matériels et comportements d'apprenants. 
Apart from its material aspect, the reality of a language centre consists in a cognitive 'space' aimed at fostering learning. Learners evolve in this space more or less independently, with the staff being present to guide them where necessary. In order to set up the best organisation possible, the staff attempt to take students' representations and motivations into account, since learning in a language centre is often a new experience for them. It is necessary to evaluate the centre to find out how its resources are perceived and used. The way the centre is organised may then be altered to encourage the emergence of strategies conducive to learning. We present here an example of the "internal" reality of a language centre, where the space created is a result of the convergence between teaching theory, material resources and learners' behaviour.

\section{INDEX}

Keywords : autonomy, evaluation, motivation, teaching resource

Mots-clés : autonomie, évaluation, motivation, ressource pédagogique

\section{AUTEURS}

\section{PETER PRINCE}

Peter Prince est maître de conférences en anglais à l'Université d'Aix-Marseille 1, où il dirige le centre d'apprentissage des langues (CALUP). Ses recherches portent sur l'acquisition et l'accès lexical en L2, ainsi que sur la motivation et l'apprentissage en semi-autonomie.

peter.prince@niv-provence.fr

\section{MARIE-NOËLLE OLIVE}

Marie-Noëlle Olive est enseignante documentaliste depuis cinq ans au CALUP (Centre

d'Apprentissage de langues de l'Université de Provence), où elle s'intéresse tout particulièrement au suivi méthodologique des étudiants au cours de leur parcours en auto-formation. Auparavant elle a exercé ses fonctions dans un lycée. À ce titre elle a initié ou participé à de nombreux projets novateurs autour du multimédia - Olympiades de physique - créations de CD-rom de physique et de sites Internet. marie-noelle.Olive@univ-provence.fr 